\title{
From conservative treatment to surgery with repeated imaging modalities in post-TAVI infective endocarditis patient
}

\author{
Busra Corekcioglu ${ }^{1}$, Gamze Babur Guler ${ }^{2}$, Serpil Ozkan ${ }^{1}$, Cagdas Topel ${ }^{1}$, Ali Demir ${ }^{1}$, \\ burak onan ${ }^{3}$, and MEHMET ERTURK ${ }^{4}$ \\ ${ }^{1}$ Mehmet Akif Ersoy Thoracic and Cardiovascular Research and Education Hospital \\ ${ }^{2}$ Mehmet Akif Ersoy Gogus Kalp Damar Cerrahisi Egitim Arastirma Hastanesi \\ ${ }^{3}$ Istanbul Mehmet Akif Ersoy Egitim Arastirma Hastanesi \\ ${ }^{4}$ MEHMET AKIF ERSOY THORASIC AND CARDIOVASCULAR SURGERY \\ TRAINING AND RESEARCH HOSPITAL
}

June 18, 2021

\begin{abstract}
Transcatheter aortic valve implantation (TAVI) is the more common choice of procedure for the severe aortic stenosis patients with high surgical risk. While the popularity of TAVI increases, TAVI related complications, such as infective endocarditis (IE) increase in number. There are no specific guidelines for TAVI-IE, so shaping a path according to patient's clinical status is more reasonable. With different advantages for each, multiple imaging modalities should be used for follow-up. In our case, repeated multiple imaging modalities were used to decide early surgery versus conservative approach by detecting periaortic pseudoaneurysm after TAVI.
\end{abstract}

\section{TITLE PAGE}

From conservative treatment to surgery with repeated imaging modalities in post-TAVI infective endocarditis patient

Busra Corekcioglu1,*, MD, Gamze Babur Guler1, MD, Serpil Ozkan2, MD, Cagdas Topel3, MD

Ali Riza Demir1, MD, Burak Onan4, MD Mehmet Erturk1, MD

1 Department of Cardiology, University of Health Sciences, Mehmet Akif Ersoy Thoracic and

Cardiovascular Surgery Center, Training and Research Hospital, Istanbul, Turkey

2 Department of Infectious Disease and Clinical Microbiology, University of Health Sciences, Mehmet Akif Ersoy Thoracic and Cardiovascular Surgery Center, Training and Resarch Hospital, Istanbul, Turkey.

3 Department of Radiology, University of Health Sciences, Mehmet Akif Ersoy Thoracic and

Cardiovascular Surgery Center, Training and Research Hospital, Istanbul, Turkey

4 Department of Cardiovascular Surgery, University of Health Sciences, Mehmet Akif Ersoy Thoracic and Cardiovascular Surgery Center, Training and Research Hospital, Istanbul, Turkey

Authors' Email addresses:

busracorekci@gmail.com, gamzebabur@hotmail.com,drserpilozkan@yahoo.co.uk, cgdstpl@gmail.com, alirdemir1986@gmail.com, burakonan@hotmail.com, drerturk@gmail.com 
* Corresponding author:

Busra Corekcioglu, MD

Department of Cardiology, University of Health Sciences, Mehmet Akif Ersoy Thoracic and

Cardiovascular Surgery Center, Training and Research Hospital, Istanbul, Turkey

Istasyon Mahallesi, Turgut Ozal Bulvarı No:11 Kucukcekmece/Istanbul, Turkey

e-mail: busracorekci@gmail.com

Phone number: +905362063699

Fax number: +902124719494

Running Head: Post-TAVI infective endocarditis

Keywords: transcatheter aortic valve implantation, infective endocarditis, multimodality imaging

Count of Words: 1736

\section{ABSTRACT}

Transcatheter aortic valve implantation (TAVI) is the more common choice of procedure for the severe aortic stenosis patients with high surgical risk. While the popularity of TAVI increases, TAVI related complications, such as infective endocarditis (IE) increase in number. There are no specific guidelines for TAVI-IE, so shaping a path according to patient's clinical status is more reasonable. With different advantages for each, multiple imaging modalities should be used for follow-up. In our case, repeated multiple imaging modalities were used to decide early surgery versus conservative approach by detecting periaortic pseudoaneurysm after TAVI.

\section{MANUSCRIPT}

\section{INTRODUCTION}

Transcatheter aortic valve implantation (TAVI) is the preferred procedure among severe aortic stenosis patients with high surgical risk and comorbidities. While the elderly population grows in number, popularity of TAVI and demand for it rises. Consequently, probability of complications caused by TAVI increases. When compared to other TAVI related complications infective endocarditis is rarely seen with incidence of $0,2 \%$ $3,1 \%$ at 1 year post implantation (1) With the increase of TAVI indications, post TAVI infective endocarditis (TAVI-IE) is a subject that should be experienced. However, TAVI-IE requires expertise in diagnosis and treatment. In this report, we present a 75-year-old TAVI patient with postprocedural infective endocarditis.

\section{CASE PRESENTATION}

A 75-year-old patient with history of coronary artery bypass grafting (CABG) underwent TAVI. On the postop 2nd day, a high fever was noticed and after two sets of blood cultures were taken, intravenous antibiotic therapy was started. Transthoracic echocardiography (TTE) showed no vegetation or mass formation, so transoesophageal echocardiography (TOE) was planned. TOE images also showed no vegetation, paravalvular leakage (PVL) or perivalvular complication however a periaortic soft tissue thickening was observed (Video 1,2) In order to diagnose peri-graft infection a computed tomography (CT) scan was performed. Thickening of right coronary cusp of prosthetic aortic valve raised suspicion for vegetation formation. While the patient was taking appropriate antibiotic therapy, routine TTE controls indicated periaortic soft tissue thickening but no vegetations could be seen. After two weeks of appropriate antibiotic therapy, control CT scan displayed small milimetric vegetations on TAVI stent struts (Figure 1A, 1B). While the patient was under treatment, his blood work showed a regression of infection markers and he was asymptomatic, therefore it was decided to continue to follow the patient with the conservative approach by the Infective Endocarditis Team. A week after the second CT scan high fever was detected again and in order to diagnose new complications resulting from infective endocarditis a control TOE was planned. TOE images (video 3,4 ) 
showed pseudoaneurysm at periaortic site therefore a surgical approach was obligatory for the patient rather than a conservative one. After the preop preparations re-do aortic valve replacement was done successfully. There were no complications after the surgery and control imaging proved a normo-functioning bioprosthetic aortic valve.

\section{DISCUSSION}

According to American Heart Association (AHA) 2020 guideline transfemoral TAVI is recommended for the patients who are 80 years of age or older or younger patients whose life expectancy is less than 10 years and patients of any age whose surgical risk is high and whose post-TAVI life expectancy is more than 12 months (2) Even though TAVI is considered to be the less invasive approach compared to surgical aortic valve replacement (SAVR), it still has a considerable amount of complications that may affect the outcome. These complications include stroke, life threatening bleeding, coronary artery obstruction, aortic root rupture, acute kidney injury, moderate or severe prosthetic valve regurgitation, prosthetic valve endocarditis or prosthetic valve thrombosis (3) In this case we presented an early prosthetic valve endocarditis (PVE) as a complication of TAVI, its diagnosis and our clinical approach.

A systematic review from Khan et al. (4) showed that the mean incidence of post-TAVI IE is 3,25\%. and showed a high mortality rate with the mean incidence of in-hospital mortality as $29.5 \%$. Post TAVI-IE incidence was detected to be similar to post SAVR-IE in many recent trials (5). However, Post TAVI IEassociated mortality is significantly higher compared to IE after native or prosthetic valve surgery (6). The elderly patient population with high comorbidities seems reasonable to explain why the post TAVI-IE mortality was the highest. Renal impairment, moderate to severe PVL, orotracheal intubation, self-expandable valve system, malposed valve, excessive manipulation, and transapical approach have been reported as predictors of TAVI-IE in various studies $(5,7)$.

For diagnosis European Society of Cardiology (ESC) suggests a combined approach to PVE using multiple imaging modalities such as TTE, TEE, computed tomography (CT), positron emission tomography (PET) or single-photon emission computed tomography (SPECT) (8) As we implemented with this case of post - TAVI IE, CT is essential while diagnosing perivalvular complications and can give a more detailed and accurate anatomical information compared to TTE or TOE in PVE. (9) Excessive metal component of TAVI valve and presence of remnant calcified aortic valve cause difficulties in echocardiographic evaluation (7). Therefore, repeated echocardiographic evaluations and / or combined with other imaging modalities in diagnosis of PVE is of the utmost importance in this patient group.

For now, there is no special guidelines or recommendations for the management of TAVI IE. Parenteral antibiotic therapy is still deemed to be the first step of the management of TAVI IE (10). The group of patients who undergo TAVI procedure have a higher risk profile and more comorbidities compared to other groups with native valve endocarditis or post-SAVR endocarditis. For this reason, overall data shows that TAVI IE patients are less frequently operated on when compared to the other IE patients (1) Anatomical complications caused by peri-annular extension of the infective process such as involvement of mitral valve and perforation of anterior mitral valve leaflet or development of periaortic pseudoaneurysm (10) require immediate surgical intervention. In the absence of these mechanical complications, decompensated heart failure, uncontrolled infection or peripheric embolism, it is found to be reasonable to keep a conservative approach with parenteral antibiotic therapy (1) The infective endocarditis team has an extremely important function in TAVI -IE with individualized treatment management choices. We initially chose to continue with the conservative path in our patient for several reasons: 1- The general condition of the patient was stable and fever control was achieved with antibiotic treatment 2- Infectious laboratory values decreased significantly at the beginning of the therapy 3 - No paravalvular complications were seen at the first imaging (echocardiography/CT) evaluations. With different advantages for each, multiple imaging modalities should be used for follow-up. The crucial point in the management of this patient was the detection of a silent pseudoaneurysm before the patient's clinical deterioration. From this point on, we believe that timing of surgery without clinical and laboratory deterioration is the most important step in patient survival. In order to decide on conservative or invasive approach for patients with TAVI-IE, a close follow-up of symptoms and 
clinical status with the help of different and repeated imaging modalities is required.

\section{CONCLUSION}

TAVI IE is a rare post-procedural complication, however it is possible to see a rise of the number of TAVI IE as TAVI becomes a more common procedure among elderly and even younger populations whose longer life expectancy may result in long term complications including IE. There are no specific guidelines for TAVI IE but recommendations from systematic reviews. There is need for more research to determine whether surgical or conservative approach is beneficial for these patients. As this population of patients are fragile and have high surgical risk profile, determination of which conservative or surgical method is the most appropriate with close follow-up and repeated imaging methods seems more sensible.

\section{Figure Legends}

Figure 1: Small milimetric vegetations (red arrows) on TAVI stent struts at CT scan (Figure 1A: CT scan coronal plane, Figure 1B: CT scan axial plane)

\section{Video Legends}

Video 1: Peri-aortic soft tissue thickening and hyperechogenity (mid esophageal long axis TOE view - red arrowhead)

Video 2: Peri-aortic thickening (mid esophageal long axis view with colour Doppler - red arrowhead)

Video 3: Peri-aortic pseudoaneurysm (mid esophageal long axis TOE view - red arrowhead)

Video 4: Peri-aortic pseudoaneurysm (mid esophageal long axis view with colour Doppler -red arrowhead)

\section{REFERENCES}

(1) Harding D, Cahill TJ, Redwood SR, et al. Heart 2020; 106: 493-498

(2) Otto, C. M., Nishimura, R. A., Bonow, R. O., Carabello, B. A., Erwin, J. P., Gentile, F., Jneid, H., Krieger, E. V., Mack, M., McLeod, C., O'Gara, P. T., Rigolin, V. H., Sundt, T. M., Thompson, A., \& Toly, C. (2021). 2020 ACC/AHA Guideline for the Management of Patients With Valvular Heart Disease: A Report of the American College of Cardiology/American Heart Association Joint Committee on Clinical Practice Guidelines. Circulation, 143(5).

(3) Möllmann H, Kim W, Kempfert J, et al. Complications of transcatheter aortic valve implantation (TAVI): how to avoid and treat them. Heart 2015;101:900-908.

(4) Khan A., Aslam A., Satti K.N., Ashiq S. Infective endocarditis post-transcatheter aortic valve implantation (TAVI), microbiological profile and clinical outcomes: A systematic review. PLoS ONE. 2020;15:e225077. doi: 10.1371/journal.pone.0225077.

(5) Henrik Bjursten, Magnus Rasmussen, Shahab Nozohoor , Mattias Götberg, Lars Olaison, Andreas Rück, Sigurdur Ragnarsson. Infective endocarditis after transcatheter aortic valve implantation: a nationwide study. Eur Heart J 2019 Oct 14;40(39):3263-3269

(6) Amat-Santos IJ, Messika-Zeitoun D, Eltchaninoff H, et al. Infective endocarditis following transcatheter aortic valve implantation: results from a large multicenter registry. Circulation 2015; 131: 1566-1574.

(7) Emmanouil Chourdakis, Ioanna Koniari, George Hahalis, Nicholas G Kounis, Karl Eugen Hauptmann.Endocarditis after transcatheter aortic valve implantation: a current assessment. J Geriatr Cardiol 2018; 15: 61-65.

(8) Gilbert Habib, Patrizio Lancellotti, Manuel J Antunes, Maria Grazia Bongiorni, Jean-Paul Casalta, Francesco Del Zotti, Raluca Dulgheru, Gebrine El Khoury, Paola Anna Erba, Bernard Iung, Jose M Miro, Barbara J Mulder, Edyta Plonska-Gosciniak, Susanna Price, Jolien Roos-Hesselink, Ulrika Snygg-Martin, Franck Thuny, Pilar Tornos Mas, Isidre Vilacosta, Jose Luis Zamorano, ESC Scientific Document Group, 2015 ESC 
Guidelines for the management of infective endocarditis: The Task Force for the Management of Infective Endocarditis of the European Society of Cardiology (ESC) Endorsed by: European Association for CardioThoracic Surgery (EACTS), the European Association of Nuclear Medicine (EANM), European Heart Journal, Volume 36, Issue 44, 21 November 2015, Pages 3075-3128, https://doi.org/10.1093/eurheartj/ehv319

(9) Feuchter GM, Stolzmann P, Dichlt W, et al. Multislice computed tomograhy in infective endocarditis: comparison with transesophageal echocardiography and intraoperative findings. J Am Coll Cardiol 2009; $53: 436-44$

(10) De Palo M, Scicchitano P, Malvindi PG, Paparella D. Endocarditis in Patients with Aortic Valve Prosthesis: Comparison between Surgical and Transcatheter Prosthesis. Antibiotics (Basel). 2021 Jan 6;10(1):50. doi: 10.3390/antibiotics10010050. PMID: 33419074; PMCID: PMC7825452.
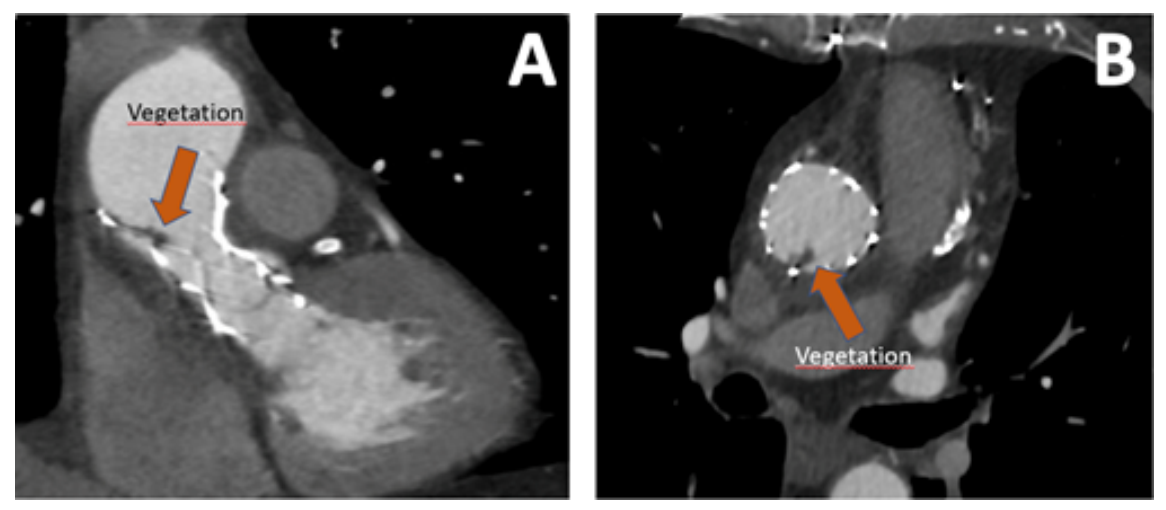